\title{
DOCENCIA UNIVERSITARIA Y CONOCIMIENTOS EN TORNO AL GÉNERO. RESISTENCIAS, CREENCIAS Y PREJUICIOS ${ }^{1}$
}

\author{
Pilar Ballarín Domingo \\ ballarin@ugr.es \\ Universidad de Granada
}

Recibido: 12-02-2013

Aceptado: 23-03-2013

\begin{abstract}
Resumen
El conocimiento producido desde el marco de los Estudios de las Mujeres, Feministas y de Género, en diversos ámbitos del saber, ha sido muy notable en España en las últimas décadas. Su impacto en los espacios de investigación es indiscutible e igualmente su alcance a la agenda política. Sin embargo, los conocimientos que se transmiten y difunden en las universidades españolas, por lo general, se mantienen impermeables a las aportaciones señaladas. En este artículo se presentan resultados de una reciente investigación, en la que se ha indagado sobre las creencias y prejuicios que perviven en el profesorado y que ayudan a comprender los tipos y niveles de resistencias que se producen ante las aportaciones del feminismo académico en los diversos campos de conocimiento.
\end{abstract}

Palabras Clave: Universidad, España, profesorado, docencia, resistencias, creencias, prejuicios, Estudios de las Mujeres, feminismo, género.

\begin{abstract}
The knowledge obtained from the Women, Feminists and Gender Studies Framework, in different fields of knowledge, has been remarkable in Spain in the last decades. It is undeniable its impact in different research fields as well as its implications in the political agenda. Nevertheless, the knowledge transmitted and spread in Spanish Universities remained constant to the already mentioned contributions. In this paper, we can see the results obtained from a recent research, in which different beliefs and prejudices still present in the teaching profession have been studied. These results help to understand the different types and levels of resistance existing in several fields of knowledge, in order to avoid these contributions from the academic feminism.
\end{abstract}

Key Words: University, Spain, teaching profession, teaching, resistances, beliefs, prejudices, Women's Studies, feminism, gender.

\footnotetext{
${ }^{1}$ Este artículo se inscribe en el marco del Proyecto "Evaluación de la incidencia de los saberes de las Mujeres, Feministas y de Género en la docencia universitaria" Proyecto I+D+I. Plan Nacional. Ministerio de Trabajo y Asuntos Sociales. Instituto de la Mujer. Acción Estratégica sobre Fomento de Igualdad de Oportunidades entre mujeres y hombres 2007. No 074/07 Investigadoras: M ${ }^{\mathrm{a}}$ Ángeles Gálvez, Vicenta

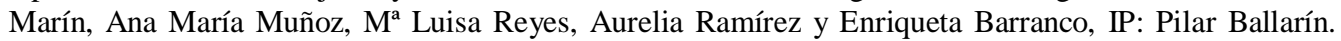
Informe completo depositado en la Subdirección de Estudios del Instituto de la Mujer.
} 


\section{Introducción}

Son muchos los trabajos que informan del impulso y desarrollo de los Estudios de las Mujeres, Feministas y de Género, también denominado feminismo académico, en las últimas décadas (Ballarín, Gallego y Martínez, 1995; Ortiz, Birriel y Marín, 1998; Ortiz et al., 1999; Ortiz, 2004; Maquieira et al., 2005; Martínez, 2006). El fruto de las investigaciones en Estudios de las Mujeres, Feministas y de Género y su alcance a las distintas áreas de conocimiento, aunque está siendo lento, comienza a apreciarse sobre todo en las áreas de Ciencias Sociales y Humanidades en las que ya son raros los eventos académicos en que no haya aportaciones desde la perspectiva de género, y pocas son las Revistas científicas de estas áreas que no hayan dedicado a estas cuestiones algún número monográfico. Sin embargo, como se desprendía del balance de 25 años que nos ofrece la obra Democracia, Feminismo y Universidad en el siglo XXI (Maquieira et al., 2005), esta aparente aceptación de los nuevos saberes, no estaba alcanzan do a las aulas universitarias, es decir, a los conocimientos que en ellas se transmiten y difunden. La docencia universitaria, salvo en los contados casos en que existe alguna asignatura optativa específica $^{2}$, se seguía desarrollando ajena a la producción del feminismo académico y sin consideración alguna a los análisis de género que puedan permitir al alumnado comprender la construcción de realidades sociales y científicas desiguales en función del sexo.

Las diversas normas y leyes desarrolladas por los últimos Gobiernos socialistas para promover mayor igualdad y, en especial la Ley Orgánica 3/2007, de 22 de marzo, para la igualdad efectiva de hombres y mujeres ${ }^{3}$, que ha venido a reconocer el valor de estos conocimientos en los que tales leyes se fundamentan, no parecen haber tenido el impacto esperado. Las universidades, inmersas en el denominado proceso de implantación de los nuevos planes de estudio para la convergencia en el Espacio Europeo de Educación Superior, han centrado su atención en los aspectos organizativos más burocráticos y, a pesar del esfuerzo desarrollado, el resultado podría decirse que ha sido más formal que sustancial ${ }^{4}$.

En este contexto, podemos afirmar, que la aportación teórica y metodológica de los Estudios de las Mujeres, Feministas y de Género sigue caminando en paralelo sin llegar a impregnar los conocimientos considerados "generales". Sin duda, el techo de cristal o el laberinto que encuentran las mujeres en la universidad (Ballarín, 2005) impide no solo el

\footnotetext{
${ }^{2}$ Por lo general asignaturas optativas creadas ya en los planes de estudio, a partir de 1995.

${ }^{3}$ En el Artículo 25. La igualdad en el ámbito de la educación superior en el punto 2 se señala: "En particular, y con tal finalidad, las Administraciones públicas promoverán: a) La inclusión, en los planes de estudio en que proceda, de enseñanzas en materia de igualdad entre mujeres y hombres. b) La creación de postgrados específicos. c) La realización de estudios e investigaciones especializadas en la materia." (España, 2007: 12616-12617).

${ }^{4}$ Aunque no se disponen todavía de datos precisos, las asignaturas específicas en materia de Género se han visto reducidas en la mayoría de las Universidades.
} 
progreso de las mujeres y sus producciones intelectuales sino también que la universidad aproveche sus potencialidades. Este hecho ha sido denunciado en numerosos trabajos como por ejemplo los referidos a la posición de las mujeres en la universidad (García de León y García de Cortazar, 2001; García de Cortazar, 1997; Anguita, 2003; Álvarez, Solano, Güil, 2005; Unidad de Mujeres y Ciencia, 2007) y en ámbitos de la ciencia y tecnología (Barral, Magallón, Miqueo y Sánchez , 1999; Pérez Sedeño, 2001, 2003, 2007; Pérez Sedeño y Santesmases, 2005) y explican en gran parte esta situación. La menor presencia de las mujeres en el conjunto del profesorado universitario, su posición en las escalas más bajas y su escasa representación en los niveles de decisión académica, a pesar de la mayor paridad es, sin duda, un factor clave a la hora de explicar la limitada capacidad para incluir materias específicas en los planes de estudio. Pero también es necesario identificar dónde se encuentran, si las hay, las resistencias del profesorado a los saberes producidos desde el feminismo académico y que impiden su inclusión en los programas docentes.

Estas consideraciones motivaron el proyecto de investigación, en el que se inscribe este artículo. Partía de la idea de que la ausencia en los programas docentes de los conocimientos aportados por la investigación feminista y de género, de indudable interés científico, social y educativo, no sólo está dificultando en el alumnado universitario la comprensión de una realidad marcada por la discriminación a pesar del progreso de la igualdad sino que, por omisión, contribuye a la naturalización de las diferencias construidas y a la reproducción de los prejuicios más arraigados. Entre sus objetivos ${ }^{5}$ buscaba indagar en profundidad dónde se encuentran las resistencias que provocan, de manera invisible, la mayor o menor permeabilidad de distintos campos de conocimiento a las aportaciones procedentes de los Estudios de las Mujeres, Feministas y de Género.

La investigación, en síntesis, se trata de una indagación en profundidad a través de un estudio de caso, combinando herramientas cuantitativas y cualitativas y estableciendo puentes entre ellas, que ha hecho posible la aparición de categorías emergentes o cuestiones (issues) que pueden favorecer nuevas comprensiones a nuestro objeto de investigación. Para ello seleccionamos una universidad, como sistema acotado, como institución singular sobre la que se realiza un tipo de estudio en profundidad denominado por Stake (2006) "instrumental” porque sus características suponen un caso ilustrativo por las circunstancias que representa y las especiales características de sus programas, actores y actrices. Se trata de un estudio de caso que puede proporcionarnos riqueza de

\footnotetext{
${ }^{5}$ 1. Determinar el alcance de penetración de los conocimientos sobre las mujeres, feministas y de género en la docencia universitaria. 2. Reconocer las áreas o ámbitos más sensibles y explicar los motivos de ésta. 3. Identificar las áreas menos permeables y buscar las condiciones en que se producen sus resistencias.4. Detectar los prejuicios que impiden la incorporación de los nuevos saberes. 5. Caracterizar las creencias del alumnado sobre éstos. 6. Formular recomendaciones para la mejora de la docencia universitaria.
} 
información ${ }^{6}$ por la situación de transición en que se encuentra la Universidad de cara al proceso de adaptación al espacio europeo de educación superior.

En este caso se abordan distintos niveles de análisis de los contenidos de los programas docentes ${ }^{7}$, de las opiniones del alumnado ${ }^{8}$ y de los discursos del profesorado.

En este artículo se presenta una valoración de algunos resultados obtenidos a partir de las entrevistas en profundidad al profesorado. Se realizaron 15 entrevistas en profundidad a una muestra del profesorado de las diferentes titulaciones seleccionadas: Maestro/a de Primaria (4), Pedagogía (3), Psicología (2), Medicina (3) e Historia (3). Siguiendo criterios previstos para los siguientes estratos: Sexo (8 profesoras y 7 profesores); consideración o no de cuestiones de género en los programas (6 que tratan o dicen tratar cuestiones de género y 8 que no) nivel de experiencia (6 con 30 años o más de experiencia y 9 con menos de 20) y de 11 áreas de conocimiento diferentes. Una vez trascritas las entrevistas, para su análisis hemos utilizado el programa Nudist* Vivo (Nonnumerical Unstructured Data with powerfül processes of Indexing Searching and Theorizing), codificando e indexando los discursos.

En estas entrevistas se perseguía detectar las resistencias, creencias y prejuicios de quienes están involucrados en el desarrollo de diversas asignaturas o materias, considerando los significados e interpretaciones individuales, buscando la comprensión de sus experiencias e identificando el sentido que dan a los saberes que nos ocupan ${ }^{9}$.

El análisis que se presenta se encuentra estructurado en cuatro bloques o apartados, 1. Desconocimiento y no reconocimiento de la producción científica sobre las mujeres, 2. Conciencia sin ciencia, 3. Efectos de una producción feminizada y 4. Transversalizar desde las conciencias. Este orden de exposición busca dar razón progresiva de las conclusiones con que se cierra este artículo.

\footnotetext{
${ }^{6}$ No presentamos aquí sus características para no interferir en el anonimato que debe caracterizar este tipo de investigación.

${ }^{7}$ Recopilación y análisis documental: Revisión de los programas de todas las asignaturas de las titulaciones de Magisterio de Educación Primaria (diplomatura) y licenciaturas de Pedagogía, Medicina, Psicología e Historia, para identificar las referencias explícitas a cuestiones relativas a las mujeres, género y feminismo en el título, objetivos, contenidos y referencias bibliográficas.

${ }^{8}$ Diseño, construcción, aplicación y tratamiento estadístico de los datos de un cuestionario dirigido al alumnado de último curso de las titulaciones señaladas. Preguntas semi-estructuradas con el fin de a) afinar el tratamiento de cuestiones relativas a las mujeres, género y feminismo en los programas, b) obtener información sobre el tipo de tratamiento de dichas cuestiones, c) identificar sus creencias y prejuicios sobre estos temas y d) obtener datos diferenciados en función de las variables independientes y dependientes que se especificaron en el diseño

${ }^{9}$ Hay que señalar que, en todos los casos, la aceptación de las personas entrevistadas para colaborar fue rápida y explícita y agradecemos muy sinceramente la atención que nos prestaron. La constante aparición de puntos suspensivos “..." en los textos trascritos de nuestras entrevistas es debida, en muchas ocasiones, a razones metodológicas, por la necesidad de omitir referencias que identifiquen a personas o lugares, etc.
} 


\section{Desconocimiento y no reconocimiento de la producción científica sobre las mujeres}

A través del análisis de las entrevistas realizadas se pueden detectar tres perfiles diferenciados, según la consideración que muestran hacia los conocimientos producidos en el marco de los Estudios de las Mujeres, Feministas y de Género: a) quienes muestran desconocimiento de las aportaciones científicas del feminismo académico en su ámbito científico, b) quienes conociendo ciertas aportaciones no las reconocen como científicas y c) quienes las reconocen y muestran algún compromiso con la inclusión de tales conocimientos en la docencia. Sólo el último de los perfiles es mayoritariamente de profesoras ya que el único profesor que muestra cierto reconocimiento a éstas aportaciones no muestra compromiso en su docencia. No se observan, sin embargo, en los dos primeros perfiles en los que se sitúa la mayoría de las personas entrevistas, diferencias en los discursos de profesores y profesoras, por lo que nuestro análisis nos lleva a pensar, en este caso, que creencias y prejuicios actúan indiferenciadamente en unos y otras.

La posición mayoritaria del profesorado entrevistado muestra un desconocimiento notable de la producción científica que, en las últimas décadas, ha buscado explicar a las mujeres, la génesis de las discriminaciones que perviven, la construcción social y científica de las diferencias, etc., desde los distintos campos del saber. Este desconocimiento es de tal nivel que está lejos de considerarse un déficit por sus protagonistas. Así puede apreciarse en las afirmaciones de algunos profesores y profesoras que imparten asignaturas en las que las aportaciones desde los Estudios de las Mujeres, Feministas y de Género, viene siendo abundante y relevante.

Desde esta posición hay quienes muestran cierto interés, de carácter personal, que les lleva a reconocer la necesidad de ampliar su saber. La preocupación por lo propio dirige la respuesta de quien afirma: "Yo creo que ahí habría que investigar bastante, [...] no lo he investigado, pero sí, si me preocupa ese tema, me preocupan mis hijos, [...]" (Entrevista 10; Párrafo 36). La apuesta por "habría que investigar" implica el desconocimiento de lo ya investigado, aunque en un caso el interés por la indagación se dirige hacía la ausencia de cuestiones relativas a las mujeres en sus propios programas docentes: "Tenemos que investigar qué es lo que está pasando para que, efectivamente, eso no siga siendo así" (Entrevista 7, Párrafo 30).

Este desconocimiento hace que tratar temas relativos a las mujeres y al género, en los programas docentes, dependa - como ya se ha dicho- del interés personal. Así lo afirman también las entrevistadas más comprometidas al referirse a las motivaciones del profesorado para introducir explicaciones pertinentes en los programas docentes; “[...] yo creo que... depende de que haya personas que hayan querido meterlo, tanto porque tengan una experiencia previa o porque tengan un interés general sobre el tema y se lo hayan querido preparar" (Entrevista 13, Párrafo, 30). No obstante, en opinión de una de éstas entrevistadas, no es la formación la que precede a la docencia y se vierte en ella, sino que 
depende del interés personal para que la formación se desarrolle: "Lo primero creo que es la motivación y el incentivo personal a integrarlo, y a partir de ahí la formación en ese terreno" (Entrevista 15, Párrafo 21).

En el perfil que definimos como de "no reconocimiento" también se sitúan quienes en sus discursos manifiestan ocuparse de algunas cuestiones relativas al saber sobre las mujeres, como una opción personal pero, a diferencia del grupo anterior, conociendo la existencia de dichos saberes, no los reconocen como conocimiento relevante del que ocuparse en la docencia. De nuevo, el interés personal es el que dirige a este grupo, cuando presta cierta atención, a ocuparse, generalmente, de cuestiones relacionadas con la violencia de género:

"La verdad, que no es una temática en la que yo haya profundizado mucho, pero... me gusta ver artículos, me gusta ver en libros... que toque el tema de género, en general... la violencia de género. Bueno,... y en general un poco el papel de las mujeres en la sociedad actual... Entonces,... quizá no tanto a nivel teórico, histórico, sociológico en general, cuanto en colectivos concretos. Que ya digo que [...] abordo en general la temática esta, pero [...]" (Entrevista 11, Párrafo 21).

Hay quién, por casualidad, ha descubierto el hecho diferencial de "ser mujer" en el espacio de la investigación, pero ello no le implica cambios en sus programas docentes. Así muestra un profesor su reciente interés: "Últimamente sí porque hemos topado ocasionalmente con una personaje [...] que era mujer y que, precisamente por ser mujer, pues tuvo muchas dificultades y recientemente me van interesando más estos temas que antes, ya te digo, normalmente no incidían directamente en mi trabajo" (Entrevista 3, Párrafo 20).

En algunos casos dicen reconocer las investigaciones desde el feminismo y su importancia, como quien afirma: "Por supuesto que tienen incidencia. No estoy yo personalmente puesta a la última en este tipo de investigaciones, pero si he leído mucho a Simone de Beauvoir, queda ya lejana, pero es uno de los pilares, etc." (Entrevista 8, Párrafo 9)". Pero la ambigüedad y retórica tímida e imprecisa, en otros casos, lleva más a pensar en la intención de ofrecer una opinión "políticamente correcta" que de una clara convicción, como mostramos a continuación:

“... sí, en el sentido de que están aportando una nueva visión,... enriquecedora, de lo que es la trayectoria [...] laboral, por ejemplo, en el campo de [...] ¿no? En ese sentido, ... digamos, estamos desenterrando,... ambigüedades, estamos desenterrando, digamos, aspectos que han podido entrar en contradicción con lo que realmente la sociedad exige, o lo que la sociedad debe de tener ¿no? que es esa equidad, es esa justicia social y esos... aspectos que consideramos oportuno que la formación de los futuros [...] profesionales de [...] o de cualquier otra disciplina tenga en consideración porque... realmente lo que se pretende es enriquecer y... complementar las labores y funciones que desempeña cada uno de los miembros por parte de la sociedad, ¿no? [...]” (Entrevista 12, Párrafo 21). 
Sólo las entrevistadas que se han ocupado en investigar sobre estos temas en el ámbito de su especialidad se muestran comprometidas con la inclusión de dichos conocimientos en su docencia, al considerar que "Se han hecho aportaciones muy, muy importantes sobre todo en los últimos tiempos [...]" (Entrevista 1, Párrafo 24). "En el mío en concreto -refiriéndose a su ámbito de conocimiento- sí [ha habido aportación], he aprendido muchísimo y desde luego me queda todavía mucho por aprender [...]" (Entrevista 2, Párrafo 33). Aunque también hay en este grupo quienes, siendo proclives a reconocer las aportaciones que nos ocupan, negarán la teoría feminista como marco referencial de éstas aportaciones, como veremos posteriormente.

La necesidad de una apuesta docente compleja que, por serlo encuentra dificultades, se manifiesta por una entrevistada: “[...] porque no es... fácil en contra de lo que parezca, no basta con... coger mujeres y meterlas en un tema y ya está todo solucionado, eso hay que abordarlo desde una perspectiva muy metodológica,... conceptual etc., bastante difícil... que genera siempre polémica y debate" (Entrevista 2, Párrafo 33).

La polémica y el debate se esgrime también por otros ya que como señala una entrevistada “[...] es algo que les toca muy de cerca, entonces el debate es mucho más caliente cuando trabajamos estas cosas en clase [...]" (Entrevista 15, Párrafo 51). La evitación de la polémica, aunque no siempre explícita, está presente y pone de manifiesto cómo el valor formativo de la controversia no se considera de interés cuando confronta las relaciones desiguales entre hombres-mujeres.

\section{Conciencia sin ciencia}

Como hemos señalado, lo más común y significativo, tanto entre profesores como profesoras -a excepción de las escasas personas más comprometidas- es hacer depender la inclusión o no de contenidos explicativos sobre las cuestiones que nos ocupan del interés que personalmente les despiertan. De este modo, lo que van a denominar "conciencia", en sus discursos, se relaciona más con la "sensibilidad" que con la "razón".

Al referirse a su actividad docente el profesorado muestra con claridad que no es fruto del conocimiento científico lo que le inspira considerar a las mujeres en su docencia. Como dice un entrevistado "[...] es un tema... al que sí soy muy sensible, porque me interesan las personas, todas" (Entrevista 10; Párrafo 39). Otro añade "Tienen incidencia en mí, particularmente porque desarrollamos, o intento tener una sensibilidad abierta a este tipo de cuestiones" (Entrevista 7, Párrafo 18). Lo que estos profesores denominan "conciencia" no requiere de la ciencia. La "conciencia" que se esgrime constituye una especie de sensibilización, producto de la experiencia empírica, que se produce de forma espontánea y en la que la ciencia no tiene cabida. 
"Ellos -los alumnos- tienen que desarrollar una conciencia con relación a estos temas, como con otros, o por lo menos mantenerla, si no la han adquirido... Yo creo, de todas formas, que a nivel de trato, existe un trato, desde mi punto de vista, por lo menos yo lo intento así, un trato igualitario... Entonces ¿eso se resuelve generando una conciencia o se resuelve metiendo determinados epígrafes en un programa? O resulta que... si tenemos esos epígrafes y damos esas explicaciones, y sin embargo, esa conciencia... se sigue manteniendo" (Entrevista 7, Párrafo 30).

En otro sentido, pero sin recurrir al conocimiento, una profesora dice: “... no es necesario preparar un tema de este tipo para encontrarte con los problemas que las mujeres, todas, nos encontramos desde la mañana hasta la noche [...]" (Entrevista 8, Párrafo 7).

Ser mujer también parece ser, para algunos entrevistados varones, garantía de conocimiento, al tiempo que se adscriben sexualmente éstos saberes. "No, particularmente no doy ninguna asignatura, incluso en [...], que haya un capítulo en mente sobre estudios de género, lo da siempre una mujer. [...] siempre pretendemos que los temas los den aquellas personas que sepan más o que han trabajado en el tema" (Entrevista 3, Párrafos 12 y 16$)$.

Las ideas expresadas llevan, por lo común, a que las razones en torno a las desigualdades entre hombres y mujeres formen parte de un totum revolutum de desigualdades sociales, en las que no parece existir diversidad en sus génesis, y que expresa así un entrevistado:

"[...] Y luego quizá desde visión de colectivos un tanto desfavorecidos, pero que en cierta manera el hecho de ser mujer les añade algo más. Es decir, a esos grados de marginación, de exclusión a que pueden llegar determinadas mujeres, como puede ser el tema de la prostitución, por ejemplo, el tema de los "sin hogar", que se llamarían hoy, cada vez están incorporándose más mujeres a este colectivo, en concreto a los de "sin hogar". Y bueno, les añade el hecho de la marginación, la exclusión que llevarían en común hombres y mujeres, pues a estas les añade también mayor marginación, exclusión, por el hecho de ser mujer" (Entrevista 11, Párrafo 21).

En consecuencia, las explicaciones que se trasladan al alumnado, aunque no se duda sean bien intencionadas, muestran muy escasos conocimientos y sensibilidades, cuanto menos muy peculiares:

"[...] eso yo lo he trabajado mucho en clase, y les ponía ejemplos: ella está a mil kilómetros, el a otros mil kilómetros, tienen un trabajo porque ninguno de los dos es seguro y tal... ¿Pues no sería mejor, que alguno de los dos pudiera tener un trabajohobby, o un trabajo que se pudiera realizar de otra manera, y pudieran compartir, etc. Como ves es un tema que yo no he trabajado, pero al que sí soy muy sensible [...]" (Entrevista 10, Párrafo 39). 
La violencia de género y las situaciones de discriminación son los aspectos que, según se desprende de las informaciones, han despertado la "sensibilidad" del profesorado, podría decirse que esta conciencia lleva a rellenar de mujeres-víctimas el escaso espacio que se les dedica en los programas docentes y que no busca ser explicativo sino sensibilizador.

“[...] vemos cómo abordar metodológicamente problemáticas sociales, [...] hay uno de los seminarios que es sobre [...] y entonces abordamos el problema de la violencia de género y los estudios que desde [...] se hacen para, un poco saber más sobre este fenómeno y cómo intervenir en él. Y en la asignatura de [...] pues ya pasamos, damos un paso más y diseñamos proyectos, pues para atender la problemática. Sobre todo, lo que trabajamos es violencia de género, en tanto como afecta a las mujeres como a los hijos, desde la perspectiva también de los niños, es lo que más trabajamos" (Entrevista 6, Párrafo 23).

"[...] tenemos unos materiales de prácticas que son ya muy numerosos y abundantes donde recuerdo que tenemos algunos muy interesantes sobre violencia, sobre violencia doméstica, en cuestión... bueno... pues, la legislación de la época o la tolerancia que había en el tema de los celos y del adulterio, testimonios de mujeres maltratadas y cosas por el estilo, sí hay alguna. Pero no, no, la asignatura no esta enfocada con una idea de género, sino únicamente con una visión generalista como corresponde a la asignatura" (Entrevista 1, Párrafo 16).

En el tratamiento de esta candente cuestión, que centra la atención de muchos entrevistados y entrevistadas, queda patente cómo la desinformación puede convertirse, en algunos casos, en deformante a falta de conocimiento adecuado. "Muchas veces los trabajos que hemos realizado en las asignaturas han ido en esa línea, de violencia de género, en la línea de hacia la mujer. Sin embargo yo he tenido trabajos hechos por mujeres, muy buenos, de violencia hacia el hombre" (Entrevista 10, Párrafo 36).

Quienes en su discurso parecen admitir la existencia de aportaciones científicas, sin embargo, justifican la ausencia de explicaciones pertinentes en su docencia porque consideran que se trata de una particular "conciencia" individual, de lo social, que incide en el desarrollo de actitudes y valores alejadas de su campo científico.

Afirmar que se tratan estas cuestiones "Sobre todo... cuando estamos intentando, [...] abordar problemáticas sociales" (Entrevista 6, Párrafo 31), es una idea generalmente implícita que expresan la mayoría de los entrevistados y entrevistadas y que les lleva, como hemos señalado, a tratar cuestiones relacionadas con la violencia de género o discriminaciones muy evidentes con ánimo de denuncia más que de explicación racional desde su ámbito científico. 


\section{Efectos de una producción feminizada}

Los principales obstáculos detectados entre el profesorado para reconocer las aportaciones científicas de los Estudios de las Mujeres, Feministas y de Género, podemos situarlos en dos ámbitos estrechamente relacionados. Por una parte la pervivencia entre el profesorado de una anacrónica concepción de la ciencia y, por otra, la feminización de estos nuevos conocimientos.

Quienes consideran la ciencia como neutral, impersonal e impermeable a los cambios sociales, que son mayoría entre el profesorado entrevistado, encuentran grandes obstáculos para aceptar que el conocimiento desarrollado para explicar a las mujeres tiene algo que ver con esa concepción de la "razón neutra", entendiendo estas aportaciones como cuestiones más próximas a sentimientos, emociones, o ideológicas. Sólo parecen liberarse de estas ideas quienes se han cuestionado ese modelo de ciencia. Esta cuestión, como se ha dicho, es inseparable de dónde se sitúa el origen de los conocimientos explicativos en torno al género.

Ha sido relevante para el objetivo de esta investigación reconocer cómo, en los discursos del profesorado entrevistado, afloran prejuicios arraigados cuando se les interroga sobre dónde ubicar el origen de éstos nuevos conocimientos.

Si bien es cierto que los conocimientos generados desde los denominados Estudios de las Mujeres, Feministas y de Género, en los distintos ámbitos científicos, se han producido en las instituciones universitarias y de investigación, hay que explicarlos también como la respuesta académica de un movimiento cuyo compromiso colectivo sienta sus raíces en el marco de la teoría feminista, lo que explica su feminización. Este hecho hace que su mayor o menor reconocimiento dependa, mas que del valor en si mismos de los conocimientos generados, de la "masa crítica" del área correspondiente y de la pervivencia de prejuicios arraigados hacia todo lo que pueda considerarse feminista, muy presentes hasta en sectores que se consideran críticos.

En el reconocimiento del origen feminizado de estos saberes se observan muy diversos perfiles. Ante la pregunta de si son las mujeres las principales productoras de conocimiento en éste campo, hasta quienes desconocen sus resultados responden con cierta cautela, inseguridad o incomodidad, sobre todo cuando la respuesta es afirmativa, haciendo un esfuerzo para evitar que se entienda como un espacio de "exclusividad" de las investigadoras, o bien desde la consideración de que la aceptación de una autoría femenina mayoritaria devalúa el campo de conocimiento de origen.

Así, un entrevistado ante la feminización de los conocimientos en torno al género, afirma: "Sí, yo pienso que sí, que es lo habitual, no necesariamente, pero es lo habitual" (Entrevista 1, Párrafo 28), a pesar de apostillar que "no necesariamente" considera necesario excusarse más adelante afirmando que "siempre lo he visto con esta naturalidad" (Entrevista 1, Párrafo 32). 
Para el profesorado entrevistado, el reconocimiento de la autoría femenina se presenta como algo imprevisto, difícil de aceptar sin aclaraciones: "Pues... quizá en esta temática... como especialización, no soy una estudiosa en el pleno sentido, pero lo que yo he ido leyendo..., y, prioritariamente, sí, han sido más mujeres que hombres [...]" (Entrevista 11, Párrafo 25). En este caso, se apostilla que: "Aunque afortunadamente hay ya bastantes, yo diría que también hay una concienciación por parte de autores, de hombres en concreto, que van abordando la temática también" (Entrevista 11, Párrafo 27).

Con cierta ironía una informante reparte sexualmente lo que se considera ciencia "dura" de ciencia "blanda" al indicar "Pues desde esta perspectiva así, sí. Porque se pueden abordar desde una perspectiva más [...] y ahí sí están los hombres, pero desde una perspectiva más de comprensión del problema, de variables, la verdad es que, hay más mujeres -ríe-" (Entrevista 6, Párrafo 35).

Siendo de común aceptación el reconocimiento de la feminización de estos estudios, aunque con posiciones ambiguas, hay que tener en cuenta que quienes sitúan su origen fuera del correspondiente ámbito académico de conocimiento no reconocen las aportaciones y, sin embargo, quienes sí las reconocen consideran que son producto del propio ámbito científico, aunque, en ocasiones, para ello tengan que negar la feminización.

Se pronuncia claramente situando el origen fuera del área, o en paralelo a la misma, quien afirma: "No, en paralelo, dentro del campo de conocimiento no. Puede que haya dado la casualidad que algunas personas de mi ámbito se hayan preocupado por estos temas, pero no necesariamente hay vinculación, no hay una vinculación necesaria. En paralelo" (Entrevista 9, Párrafo 25). Otro caso, en el mismo sentido, dice: "En mi área de conocimiento... poco, quiero decir, en [...] relativamente poco, en paralelo a la [...] bastante más" (Entrevista 4, Párrafo 19).

Ejemplo elocuente de la escisión feminismo-ciencia nos lo ofrece una misma persona que sitúa el origen de estos conocimientos como "reivindicación" de las mujeres, pero considera que corresponden a su área cuando se valoran como científicos: "[...] ha sido una escuela [...] muy rica nacida desde la reivindicación y es lógico que la reivindicación primera sobre la mujeres la ejerzan las mujeres ¿no?” (Entrevista 1, Párrafo 32). Aunque, anteriormente, había expresado tratarse de conocimientos propios del área: "No, no, no, dentro del área de conocimiento... el área [...] ha hecho aportaciones muy, muy importantes sobre todo en los últimos tiempos a la [...] de la Mujer y ya digo, y algunas incluso hechas desde... [esta misma Universidad]. Que claro que se han aportado a la docencia, sî" (Entrevista 1, Párrafo 24).

Con una valoración similar, otra informante reconoce el origen de los conocimientos en el área: "[...] han surgido dentro, en el sentido en el que son las propias [...] las que han sacado adelante una serie de trabajos importantísimos, o sea, que no es que hayan venido de fuera.... [del área]" (Entrevista 2, Párrafo 41). Pero parece necesitar reafirmar la participación masculina para acreditar el valor científico de éstos: "No tengo una estadística de que investigadores e investigadoras que se han dedicado a estos temas y el porcentaje de investigadoras mujeres que se ocupan de [...] y el porcentaje de [...] varones que se ocupan de 
este tema. Pero me da la impresión de que son sobre todo [mujeres] y tendría que tener una estadística delante, pero [...]" (Entrevista 2, Párrafo 49).

La insistencia en que "los varones también", presente en otras entrevistas, parece querer negar lo que se desconoce al percibirlo como discriminatorio.

"Hay muchos hombres también... hay muchos hombres también. Quizás las mujeres como nos vemos dentro de ese mundo ¿no? y nos sentimos muchas veces discriminadas por cuestiones... ¿no?... pues nos preocupemos más, nos llame más la atención, pero hay muchos hombres que también, hay muchos hombres que también. De hecho, hay muchas mujeres que pasan de estos [...] totalmente. Tampoco hay vinculación necesaria entre ser hombre o ser mujer para hacer [...]" (Entrevista 9, Párrafo 29).

Se entiende que la producción en el marco del feminismo académico tiene por objetivo central a las mujeres, pero explicar a éstas se considera un conocimiento parcial que no forma parte del TODO ya definido para la transmisión docente (contenido científico neutral, impersonal e inmutable) por lo que se argumenta la imposibilidad de su inclusión en los programas de enseñanza, obviando que se trata de media humanidad: "[...] porque el programa ahora mismo no puede dar respuesta a todas las necesidades que estamos teniendo a nivel de formación [...]" (Entrevista 7, Párrafo 26).

"Bueno yo la asignatura que tengo es una asignatura genérica, de las troncales del área, y no, no lleva incluida esa perspectiva porque digamos que es el resumen de los contenidos propios [...]. Otra cosa es que dentro de cada apartado, pues bueno, siempre se puede tratar el tema de las mujeres, pero no, no, generalmente en la materias de [...] en el área a la que pertenezco generalmente esos temas se abordan dentro de la asignatura de [...]" (Entrevista 1, Párrafo 12).

Con otro enfoque, también hay quien diferencia las exigencias de atender a "lo relevante" frente a "lo insignificante":

"[...] estamos ante un problema que realmente es serio porque nosotros tenemos que competir, tal como estamos, tenemos que competir en condiciones de igualdad con países que resulta que tienen un alto componente académico, una formación académica estupenda y sin embargo, además tenemos que conseguir cumplir con esos compromisos de igualdad, de solidaridad, y lo estamos haciendo a base de no cumplir con los objetivos de competencia, que se supone que después se les exige a esos profesores, etc. y a esas personas. Entonces claro, aquí estamos generando unos antagonismos grandes" (Entrevista 7, Párrafo 52).

Para otros entrevistados es el peligro de lo que consideran "ideologización” lo que dispara sus las alarmas: "[...] hay una intencionalidad muy fuerte, es una intencionalidad de base. Hay una discusión, por ejemplo, [...] si el género entra o no entra en la [...] Yo creo que 
es una discusión más ideológica que de otro tipo, y de posicionamientos $[\ldots]$ en fin que no tiene que ver con las cuestiones que hay de fondo (Entrevista 10, Párrafo 17). Ante este peligro se defiende la neutralidad: "Yo intento siempre..., aunque se dice por ahí que no existe un componente neutral, yo lo intento siempre, si bien de un modo colateral trato cuestiones de género con las ejemplificaciones [...] Pero fuera de eso lo que no hago es una explicación de género específica, a no ser que ellos [el alumnado] me lo pidan” (Entrevista 7, Párrafo 16).

Se producen discursos muy variados entre quienes desconocen o no reconocen explicaciones científicas a la construcción de las desigualdades y piensan, como ya señalamos, que cierta conciencia basta para promover determinadas actitudes y valores. Un profesor afirma que "[...] en la asignatura siempre tengo más mujeres que hombres, de hecho me dirijo hacia el auditorio muchas veces como "vosotras", o sea que el implicarlas y el animar, por su puesto, sí, en esa perspectiva, sî" (Entrevista 5, Párrafo 18). Otros buscan alternativas de lo que consideran prácticas igualitarias:

"Yo trato... siempre... en mi asignatura,... de utilizar un discurso equitativo, de siempre, por ejemplo, hacer actuar [...] tanto a hombres y a mujeres de forma igualitaria... no igualitaria en plan dos y dos ¿no?, sino igualitaria en el sentido de... aportación de perspectivas, de reflexiones, de ideas, de conocimientos... tanto de hombres y mujeres que complementen la labor profesional mía, ¿no? [...]” (Entrevista 12, Párrafo 102).

Son significativas aquellas personas entrevistadas que achacan el hecho de no abordar cuestiones -que prefieren denominar "de género"- a su falta de formación pedagógica, pero no consideran que se trate de falta de actualización científica. Esto lleva a reclamar a "otros" lo que es responsabilidad propia.

“[...] en general el obstáculo puede venir también en que no hay una formación por parte del profesorado [...] que se ofertaran más cursos en ese sentido de formación, de reciclaje del profesorado que ya hay, para ver de que manera se puede aplicar la perspectiva de género a lo que ya enseñas, ¿no?... Quizás debería haber más cursos, no sé, de formación, de reciclaje o algo así, ¿no? [...]” (Entrevista 6, Párrafo 93).

En definitiva, la inclusión en los programas de los contenidos que nos ocupan, en los mejores casos, se concreta en añadir algún pequeño apéndice o matiz pero, en ningún caso salvo las dos entrevistadas con mayor compromiso- transformar lo existente. Una profesora afirma: "En una de las asignaturas sí, pero es solamente, es una asignatura que se titula [...] y ahí, hay un tema dedicado a la posición de las mujeres." (Entrevista 2, Párrafo 12). Y añade posteriormente: “... me parece fundamental que en los programas docentes se incluya un apartado relativo a las mujeres y específicamente dedicado a las mujeres, al menos por ahora, hasta que las cosas estén bien claras y hasta que la [...] esté en pie de igualdad con el resto de la [...]" (Entrevista 2, Párrafo 29). 
La apuesta por materias específicas, como estrategia temporal, es muy limitada. Sirva de ejemplo "[...] yo creo que indirectamente se aborda bastante, en concreto en [...] porque yo oigo de otros compañeros y compañeras y... se aborda. Pero falta quizá eso que comentábamos antes, esa base, es decir una asignatura en concreto que asiente las bases de todo esto[...]" (Entrevista 11, Párrafo 63).

\section{Transversalizar desde las conciencias}

Como ya hemos dicho anteriormente, al considerarse el saber sobre las mujeres una cuestión de actitudes y valores, no de conocimiento científico, y la igualdad entre hombres y mujeres algo ya conseguido, se niega la necesidad de inclusión de explicaciones pertinentes en los programas docentes.

"Yo pienso que en este momento quizás ya no sea necesario, porque el hecho en sí ha desbordado cualquier tipo de problema, sería absurdo defender a las mujeres en un colectivo donde el $80 \%$ son mujeres ¿no?, no tendría mucho sentido, en otras disciplinas pues, puede que sí, en otras facultades" (Entrevista 3, Párrafo 43).

Con no ser machista, es suficiente: "[...] su idea [refiriéndose al alumnado] de que somos iguales debe estar ya impresa y no hace falta que la Facultad, que tiene otras ocupaciones y transmisiones, insista en recordárselo. Lo que sí no tiene que haber es una actitud machista originaria hacia las mujeres en la Facultad, eso sí" (Entrevista 3, Párrafo 87).

En ocasiones, la responsabilidad se desvía a otras especialidades, como señala el texto anterior y como manifiesta otro informante cuando precisa quiénes deberían ocuparse de estas cuestiones “... fundamentalmente en salud y educación” (Entrevista 4, Párrafo 25); “... en otros ámbitos, en psicología y en educación, particularmente, creo que sí debería de incidirse en este aspecto, ¿no?” (Entrevista 5, Párrafo 27).

En otros casos, la educación familiar, la de otros niveles educativos, es identificada como la competente de la formación, porque en ella sitúan los informantes el necesario cambio de mentalidad que deberá producirse en el futuro ajeno a la propia responsabilidad: “[...] es un tema de educación y de una mentalidad que tendrá que ir cambiando muy lentamente, las leyes pueden ayudar pero las leyes no modifican las cosas de la noche a la mañana" (Entrevista 1, Párrafo 48); "[...] requiere un cambio social y un cambio de mentalidad y eso no se hace de la noche a la mañana eso requiere un esfuerzo muy considerable” (Entrevista 2, Párrafo 69); “[...] probablemente lo que falta es mucha educación en temas de igualdad y que la educación sea la herramienta que nos permita ir promoviendo el cambio... personal y cultural que tiene que acompañar a los cambios sociales y estructurales que son más patentes" (Entrevista 15, Párrafo 17); “[...] creo que se tiene que hacer un trabajo mucho más serio desde las propias escuelas, 
¿no? Introducir esa perspectiva de género más en serio, tan importante como la lengua y las matemáticas es eso, enseñar pues que hay igualdad en los roles [...]” (Entrevista 6, Párrafo 55).

“[...] existe una mala educación en este sentido, es decir; posiblemente en las familias hasta ahora, sobretodo en las familias españolas, han valorado enormemente la presencia de un varón, en muchas ocasiones considerando la presencia de una niña como un mal menor y esto ha hecho... pues, que la formación y lo que han dicho en el entorno los varones ha sido muy condicionado por estas ideas. Entonces... cuando eso poco a poco espero vaya cambiando, dejará de haber problemas en ese sentido" (Entrevista 3, Párrafo 83).

El mayor acuerdo entre nuestros entrevistados y entrevistadas, sensibles a cuestiones que consideran de actualidad pero que, como hemos señalado, desconocen o no reconocen como conocimientos científicos, se encuentra en la idea de transversalizar.

Afirman así que los conocimientos que nos ocupan "[...] tienen que estar de manera transversal en que todas las asignaturas y en todas las partes del programa" (Entrevista 15, Párrafo 21). Otros entrevistados dicen: “[...] con lo único que me quedo es con las transversales... Cómo hacerlo y cómo articularlo es difícil pero, a lo mejor, si se hiciera un estudio serio sobre eso se podrían encontrar vías interesantes" (Entrevista 10, Párrafo 57). "[...] la perspectiva de género debe ser en cierta medida... una óptica transversal que debe estar presente efectivamente en todas las titulaciones y esto es lo que es más difícil de conseguir ¿no? [...]”' (Entrevista 1, Párrafo 101).

Hay que recordar que, como se indicó anteriormente, algunos profesores y profesoras entienden que hay que incorporar cuestiones de género en la docencia, pero no reconocen necesidad de formación autorizada alguna para ello -salvo una excepción-. En otros casos, no se considera la necesidad de explicaciones porque se cree que la igualdad es algo ya conseguido y que hay muchas otras cuestiones que también se deberían incluir en los programas.

La transversalidad se convierte así, en los discursos analizados, en un desideratum pedagógico recurrente y casi mágico. Bastaría con tener una actitud de rechazo ante las discriminaciones y considerar como un valor la igualdad entre hombres y mujeres -que se considera ya alcanzada- para su "natural" desarrollo. Este tipo de conciencia, de la que se supone participa el conjunto del profesorado, se presume que emergerá en la actividad docente de forma espontánea impregnando al alumnado. Se trata, en definitiva de desarrollar una sensibilidad hacia cuestiones sociales, alejadas y disociadas de la razón científica que fundamenta los conocimientos profesionales.

Esta idea de transversalidad que se apoya en la defensa de que "todos podemos opinar" porque "todos y todas somos iguales" -inaceptable en cualquier ámbito científico-, se esgrime de forma perversa ante un conocimiento, desconocido, que se prejuzga impertinente, subvirtiendo la idea de igualdad, clave en sus fundamentos. 


\section{Algunas conclusiones}

Es difícil diferenciar creencias de prejuicios aunque pueden situarse a distinto nivel. Por creencias se entienden, aquellas cuestiones que se dan por ciertas sin estar comprobadas o demostradas y por prejuicios los juicios que se emiten sin fundamento o alejados de los justo o razonable, es decir, sin conocimiento previo. No obstante sería deseable esta diferenciación pues creencias y prejuicios pueden ser afrontados de diferente manera ya que las primeras podrían ser más susceptibles de corregirse con argumentos de razón, mientras los segundos sólo parecen atender a la "mostración" pues frente a la sin razón es difícil argumentar. Se enumeran, por tanto, creencias y convicciones que han ido apareciendo en los discursos analizados, al margen de si implicaban un juicio o "prejuicio" en su enunciado. En este sentido, es de interés llamar la atención sobre la saturación de algunas palabras en los discursos que nos permiten apreciar un abundante uso del verbo creer (388 frecuencias) frente al verbo pensar ( 82 frecuencias) que, al margen del uso habitual e inconsciente del verbo "creer", parece significativo de cómo se enfrentan las personas entrevistadas ante ciertas cuestiones.

De los discursos analizados se desprende que:

a) No se reconoce la existencia de un saber específico de y sobre las mujeres que dé razón y explique, desde diferentes campos científicos, la génesis de las desigualdades entre hombres y mujeres y cómo se reproducen y difunden éstas que han llegando a constituir la más persistente de las discriminaciones.

b) Lo que hay que saber sobre las mujeres y la construcción social de las relaciones de género discriminatorias es un conocimiento de tipo sociológico, que se produce de forma "natural" a través del entorno y que produce la "sensibilización" y "conciencia" hacia la discriminación.

c) No se necesita, en consecuencia, ninguna formación específica para intervenir en la docencia sobre temas de igualdad entre hombres y mujeres y no discriminación. Solo hace falta una actitud adecuada.

d) Por este mismo motivo no se considera necesario que existan asignaturas específicas ya que todo el profesorado debería transversalizar la perspectiva de "género".

La resistencia a considerar relevante el conocimiento producido parece evidente especialmente cuando se desconoce, pero cobra mayor potencia cuando a éste se añade la resistencia a la consideración de la objetividad de un conocimiento de autoría feminizada y se liga a productos más ideológicos que científicos. Estas posiciones, que son manifiestas en las resistencias a introducir materias específicas en los planes de estudio, hunden sus 
raíces en una actitud general de resistencias al cambio, a lo nuevo, a romper rutinas y a plantear controversias y más si cabe a considerar la necesidad de corregir el conocimiento androcéntrico.

El saber producido en las últimas décadas en el marco del feminismo académico, muy potente en sus resultados, se puede afirmar que no ha alcanzado reconocimiento como saber autorizado. En estos momentos cualquier profesor o profesora puede, en su docencia, pronunciarse sobre temas que desconoce o no reconoce porque los conocimientos que nos ocupan han quedado, valga la expresión, en "tierra de nadie".

Profesoras e investigadoras, en el marco de los Estudios de las Mujeres, Feministas y de Género, movidas por el compromiso de corregir el androcentrísmo científico, entendieron que la adscripción a sus áreas de conocimiento de origen favorecería una mayor transferencia de conocimientos renunciando a la pretensión de crear, en su momento, un área específica. Pero, paradójicamente, mientras no parece, por un lado, que la transferencia deseada se haya producido, al menos en el ámbito docente, por otro, el hecho de no constituir un área propia de conocimiento ha permitido la invisibilidad del conjunto de saberes autorizados que frenara aquellas intromisiones indocumentadas que no serían admisibles en otros ámbitos científicos.

\section{BIBLIOGRAFÍA}

- Álvarez, Manuela; Solano, Ana y Güil, Ana (2005): La Situación de las Mujeres en las Universidades Públicas Andaluzas. Sevilla: Consejo Económico y Social de Andalucía.

- Anguita Martínez, Rocio (2003) (coord.): Las mujeres en la Universidad de Valladolid. Valladolid: Secretariado de Publicaciones e intercambio editorial.

- Ballarín Domingo, Pilar (2005): "Mujeres en el laberinto de cristal universitario". En I. Torres Ramírez (coord.): Miradas desde la perspectiva de género. Estudios de las mujeres. Madrid: Narcea, pp. 183- 194

- Ballarín Domingo, Pilar; Gallego Méndez, M. Teresa y Martínez Benlloch, Isbel (1995): Los Estudios de las Mujeres en las Universidades españolas 1975-91. Libro Blanco. Madrid: Ministerio de Asuntos Sociales. Instituto de la Mujer.

- Barral, Ma José; Magallón, Carmen; Miqueo, Consuelo y Sánchez, Ma Dolores (1999) (eds.): Interacciones entre Ciencia y Género: Discursos y prácticas científicas. Madrid: Icaria.

- García de Cortazar, Marisa (1997): Mujeres en minoría: Una investigación sociológica sobre las catedráticas de universidad en España. Madrid: Centro de Estudios Políticos y Constitucionales. - García de León, M. Antonia y García de Cortázar, Marisa (2001): Las académicas: profesorado universitario y género. Madrid: Instituto de la Mujer. 
- España (2007): "Ley Orgánica 3/2007, de 22 de marzo, para la igualdad efectiva de hombres y mujeres", [en línea] Disponible en: http://www.boe.es/boe/dias/2007/03/23/pdfs/A1261112645.pdf [12/01/2013].

- Maquieira, Virginia et al. (2005): Democracia, feminismo y universidad en el siglo XXI. 25 aniversario del IUEM. XV Jornadas de Investigación Interdisciplinaria. Madrid: MEC, IUEM.

- Martínez López, Cándida (2006): "Las mujeres y la Universidad. Ambivalencia de su integración". En Carmen Rodríguez (ed.), Género y curriculo. Aportaciones del género al estudio y práctica del currículo. Madrid: Akal, pp. 216-225.

- Ortiz, Teresa (2004): "Consolidación y visibilidad de los Estudios de las Mujeres en España: logros y retos". En Ana M. Ruíz-Tagle (ed.): Los Estudios de las Mujeres en las Universidades andaluzas. Sevilla: Universidad de Sevilla, pp. 143-148.

- Ortiz, Teresa; Birriel, Johanna y Marín, Vicenta (1998): Universidad y Feminismo en España I. Bibliografía de Estudios de las Mujeres. Granada: Universidad de Granada.

- Ortiz, Teresa et al. (1999): Universidad y feminismo II. Situación de los Estudios de las Mujeres en los años 90. Granada: Universidad de Granada.

- Pérez Sedeño, Eulalia (2001) (ed.): Las mujeres en el sistema de ciencia y tecnología: estudio de casos. Madrid: Organización de Estados Iberoamericanos, D.L.

. (2003) (ed.): "La situación de las mujeres en el sistema educativo de ciencia y tecnología en España y en su contexto internacional". Ref: Programa de Análisis y estudios de acciones destinadas a la mejora de la Calidad de la Enseñanza Superior y de Actividades del Profesorado Universitario (S2/EA2003-0031), [en línea] en http://www.ohchr.org/Documents/Issues/CulturalRights/ConsultationEnjoyBenefits/UNESCOLASI TUACIaNDELASMUJERESENELSISTEMA.pdf [12/01/2013].

. (2007): Mujer y Ciencia. La situación de las mujeres investigadoras en el sistema español de ciencia y tecnología. Madrid: FECYT ( $2^{\circ}$ Ed. Actualizada)

- Pérez Sedeño, Eulalia y Santesmases, Mª Jesús (Coor.) (2005): Mujer y Ciencia: la Situación de las Mujeres Investigadoras en el Sistema Español de Ciencia y Tecnología. Madrid: Fundación Española para la Ciencia y la Tecnología.

- Stake, Robert E. (2006): Evaluación comprensiva y evaluación basada en estándares. Barcelona: Graó.

- Unidad de Mujeres y Ciencia. Ministerio de Educación y Ciencia. (2007): "Académicas en cifras 2007", [en línea] Disponible en: http://www.uned.es/rectoras/2007-academicas-en-cifras.pdf [12/01/2013]. 